La revue La revue pour l'histoire du CNRS

POUR L'HISTOIRE DU CNRS $\quad 17 \mid 2007$

Objectif biotechs?

\title{
Coup de soleil au CNRS...
}

La genèse du Pirdes Denis Guthleben

Denis Guthleben

\section{(2) OpenEdition}

Journals

Édition électronique

URL : https://journals.openedition.org/histoire-cnrs/2221

DOI : 10.4000/histoire-cnrs.2221

ISSN : 1955-2408

Éditeur

CNRS Éditions

Édition imprimée

Date de publication : 3 juillet 2007

ISBN : 978-2-271-06558-2

ISSN : $1298-9800$

Référence électronique

Denis Guthleben, «Coup de soleil au CNRS... », La revue pour l'histoire du CNRS [En ligne], 17 | 2007, mis en ligne le 03 juillet 2009, consulté le 20 mai 2021. URL : http://journals.openedition.org/histoire-cnrs/ 2221 ; DOI : https://doi.org/10.4000/histoire-cnrs.2221

Ce document a été généré automatiquement le 20 mai 2021.

Comité pour l'histoire du CNRS 


\title{
Coup de soleil au CNRS...
}

\author{
La genèse du Pirdes Denis Guthleben
}

Denis Guthleben

1 La décision de créer le PIRDES est adoptée le 2 juillet 1975 par le directoire du CNRS. Ce dispositif constitue une nouveauté pour l'organisme : les voies de l'interdisciplinarité y ont déjà été explorées auparavant, notamment avec les actions thématiques programmées, mais elles n'ont jamais débouché sur un programme d'une telle ampleur, auquel le Centre consacre d'emblée cinq millions de francs - ce qui correspond à près de trois millions d'euros actuels. Toutefois, si la structure est nouvelle, le contenu scientifique l'est moins. Dans ce domaine, le CNRS hérite en effet de trente ans de recherches.

Le CNRS et l'énergie solaire : une histoire ancienne

2 Les premiers travaux sur l'énergie solaire au CNRS remontent à la Libération. En 1946, le physicien Félix Trombe, qui dirige alors le laboratoire des Terres rares à Meudon, parvient à concentrer la lumière du soleil à l'aide d'un miroir de DCA - une " prise de guerre » abandonnée deux ans plus tôt par la Wehrmacht -, et obtient de hautes températures en milieu confiné. Le rapport avec les Terres rares? Rappelons que ce nom désigne un groupe de métaux aux propriétés proches qui, contrairement à leur appellation, sont très répandus dans la nature. En concentrant la lumière du soleil, Félix Trombe cherche en réalité à obtenir les températures lui permettant de faire fondre le minerai et d'en extraire des matériaux purs.

3 L'expérience s'étant révélée concluante, le physicien obtient du CNRS les crédits nécessaires pour construire un prototype de four solaire. Bien évidemment, Meudon n'est pas le site idéal pour poursuivre les travaux. Puisqu'il faut du soleil, beaucoup de soleil, Félix Trombe s'éloigne de la grisaille parisienne. Cet héliotropisme scientifique le conduit à Mont-Louis, dans les Pyrénées orientales, où le prototype voit le jour en 1947 au sein d'un nouveau laboratoire de recherches sur l'utilisation de l'énergie solaire.

4 Au cours de la décennie suivante, ce laboratoire connaît son heure de gloire en accueillant un «colloque international sur les applications thermiques de l'énergie solaire dans le domaine de la recherche et de l'industrie » du 23 au 28 juin 1958. Déjà, les applications prévues sont nombreuses : elles se rapportent «au chauffage et à la 
distillation de l'eau, à la climatisation des maisons, à la culture sous serre et à la transformation directe de l'énergie solaire en énergie électrique" ${ }^{1}$. Une nouvelle preuve, si besoin est, du formidable potentiel d'applications que renferme parfois la recherche fondamentale : dix ans plus tôt, il était uniquement question d'étudier les propriétés de certains métaux...

5 Lors du colloque, Félix Trombe présente également son projet de four solaire de 1000 $\mathrm{kW}$. Une maquette au 1/100e a été réalisée pour l'occasion, mais le directeur du laboratoire a préféré la faire présenter à l'exposition universelle qui se tient alors à Bruxelles sur le thème "Pour un monde plus humain". À défaut, les invités sont conduits sur le site retenu pour les travaux, à quelques kilomètres de là : Odeillo, qui va accueillir l'un des grands équipements les plus célèbres du CNRS. Sa construction, lancée en 1963 sous la direction de l'Aéroport de Paris et sous le contrôle des services des Ponts et Chaussées², est achevée en 1970.

Le Centre sous le feu des critiques

6 Avec l'achèvement du four d'Odeillo, le Centre dispose d'un outil exceptionnel pour les recherches sur l'énergie solaire. Il a donc une carte à jouer lorsque le premier choc pétrolier éclate (octobre 1973). Face à la facture énergétique qui explose et entraîne les effets que l'on sait sur l'économie, on lui reproche cependant de ne pas remplir son rôle avec suffisamment de célérité. Au cours de l'année suivante, les médias placent l'organisme sur la sellette. Certes, ce n'est pas la première fois et ce ne sera pas la dernière ${ }^{3}$. Mais, en 1974, les attaques sont très violentes. Elles sont d'ailleurs évoquées par le professeur Jean Lagasse devant le conseil d'administration du Centre, où il rappelle toutefois qu'» on ne saurait reprocher au CNRS la rigidité de ses structures et un temps excessif de réponse $»^{4}$.

7 "L'ampleur de la crise et ses conséquences au niveau national impliquaient la mobilisation de tout le potentiel scientifique et technique du pays", poursuit Jean Lagasse. Le Centre est d'ailleurs concerné au premier chef : l'organisme est un gros consommateur d'énergie et, pour la seule année 1974, sa facture s'est alourdie de près de quatre millions de francs selon le directeur administratif et financier Pierre Creyssel $^{5}$. L'action du CNRS a en fait déjà suivi trois voies : un inventaire des moyens, le lancement de plusieurs actions concertées sur le problème de l'énergie et la mise en place d'» une quinzaine de groupes de réflexion et de prospective sur l'énergie $»^{6}$.

8 La mise en place d'une nouvelle forme de programme scientifique est-elle déjà évoquée ? Non. Ce sont les modes traditionnels d'intervention, en particulier une action thématique "énergie» créée en collaboration avec la Délégation générale à la recherche scientifique et technique, qui ont encore la faveur de Bernard Gregory en janvier $1975^{7}$. Le directeur général du CNRS entend d'ailleurs encourager les recherches sur l'énergie solaire : avec l'installation récente d'Odeillo, «toutes les conditions sont remplies pour que puissent être faits un certain nombre de tests ». Un seul participant regrette alors l'excès de "prudence» du Centre: le directeur scientifique pour la physique, Robert Chabbal.

9 «Un programme de recherche un peu tous azimuts »

10 Robert Chabbal se souvient de la période qui a vu la création du PIRDES: "Nous formions au CNRS une petite équipe persuadée, ce qui maintenant paraît évident, de la nécessité de l'ouverture de la recherche sur le domaine socio-économique. Je pensais et je pense encore que c'est le rôle du CNRS, dans la mesure où il a su dans certains secteurs s'imposer comme l'interlocuteur de l'industrie et où il a reçu les crédits 
nécessaires à l'indépendance des laboratoires $»^{8}$. L'élaboration de ce mode d'intervention peut donc être située vers le début de 1975. On ne manquera d'ailleurs pas de faire le lien entre le programme et la création, au même moment, du département des sciences physiques pour l'ingénieur (SPI) ${ }^{9}$ : l'un et l'autre sont marqués par la même volonté d'ouverture.

11 Robert Chabbal, qui prend la direction du PIRDES, présente le programme devant ses collègues du directoire le 2 juillet $1975^{10}$. Les modalités ne sont pas encore définies: «En ce qui concerne la forme administrative qui pourrait être donnée à ce nouveau type d'action, elle n'a pas encore été précisément déterminée ». En revanche, les objectifs sont clairs : ce " programme de recherche un peu tous azimuts » devra piloter des études de météorologie, d'écologie et s'intéresser à des questions "d'aspect économique » relevant de «l'intégration de l'énergie solaire». Deux mots reviennent comme une antienne dans son exposé : « efficacité » et « souplesse ».

Paradoxalement, trois ans après la création de l'Institut national de physique nucléaire et de physique des particules (IN2P3), il est hors de question de créer une structure permanente à son image. Cela fait d'ailleurs débat lors de la réunion du directoire. Pour en prendre la mesure, il faut lire entre les lignes - laconiques et lisses -, du procèsverbal. Robert Chabbal insiste en effet «sur le fait que l'ensemble de cette affaire doit garder le nom de programme : il serait sans intérêt et contraire aux intentions de la direction du CNRS de créer un institut national, qui impliquerait en particulier une notion de très longue durée ». Le physicien nucléaire Raymond Castaing, médaille d'or du CNRS la même année, semble d'ailleurs prendre cette précision comme une critique voilée des instituts nationaux: "Une formule du type 'institut national' aurait eu sa préférence, qui aurait permis d'attirer des chercheurs confirmés, de regrouper des laboratoires du CNRS et aurait mieux montré l'effort qui était fait ». Réplique de Robert Chabbal : au moins, avec la formule choisie, «les chercheurs ne sont pas isolés dans un institut mais restent dans leur laboratoire». Mais "beaucoup de chercheurs accepteraient volontiers de changer de lieu de travail pour se consacrer à ces recherches ", renchérit Raymond Castaing, avant de laisser le dernier mot à son interlocuteur : pourquoi les faire quitter « leurs laboratoires de base à Odeillo, Marseille ou Poitiers »? Clairement, ce sont deux conceptions de la politique de recherche du Centre qui s'affrontent, l'une axée sur la solidité et la visibilité de la structure, l'autre sur les avantages de la souplesse. À l'armée, on dirait: guerre de position ou de mouvement.

Comment évaluer l'interdisciplinarité ?

Reste une question en suspens, et pas des moindres : à qui reviendra l'évaluation du programme? Cette tâche incombe traditionnellement au Comité national, mais son fonctionnement en commissions s'adapte mal au cadre interdisciplinaire. Là encore, deux conceptions s'affrontent: faire évaluer les chercheurs qui s'investissent dans le PIRDES par leur commission habituelle ou créer une commission spéciale «énergie solaire $»^{11}$. Robert Chabbal et le directeur scientifique de la chimie, Jean Cantacuzène, se montrent favorables à la première option. Le physicien Jean-Pierre Chevillot plutôt à la seconde : «Il est à craindre que les sections prises isolément ne soient pas à même d'apprécier si le projet présenté est effectivement correct. Il serait nécessaire de créer une instance dans laquelle on ait le temps de faire un examen approfondi et où seraient représentés des scientifiques venus des différentes sections ». 

indique que les sections du Comité national doivent garder la responsabilité de l'activité scientifique des équipes et des chercheurs de leur spécialité qui travaillent dans le cadre de ce projet. Il convient de trouver une formule, en ce qui concerne le groupe de personnes qui a un avis général à donner sur l'équilibre de l'ensemble du programme».

L'observation de la genèse du PIRDES renseigne en somme sur la manière dont se pense et se construit un nouveau mode d'intervention dans le domaine scientifique. Rien de linéaire, ici, mais des impulsions, des débats, des oppositions parfois, qui éclairent la recherche en mouvement. Pour preuves, les évolutions qui prennent parfois le contrepied des décisions de 1975.

À l'origine, l'option «institut national » a été écartée parce qu'elle impliquait « une notion de très longue durée ». Mais on ne peut que souligner la longévité du PIRDES, qui devient le PIRSEM (sciences pour l'énergie et les matières premières) en 1982 puis l'ECOTECH à partir de 1993. De la même manière, la mise en place d'un programme a été privilégiée pour utiliser le potentiel de laboratoires existants. Il s'agissait en quelque sorte de ne pas couler du béton. Or, dès 1978, un laboratoire d'énergie solaire est créé à Valbonne dans le cadre du programme, «en contraste, ainsi que l'annonce alors Robert Chabbal, devenu entre-temps directeur général du CNRS, avec la méthode principale de travail de ce programme qui mobilise habituellement les chercheurs sur ses objectifs propres sans création de nouveaux laboratoires ${ }^{12}$. Enfin, en ce qui concerne l'évaluation, on peut également noter que les commissions interdisciplinaires, écartées en 1975, sont instaurées en 1982. Bref, autant d'adaptations sur lesquelles il importera de revenir et qui montrent que le premier objectif du programme, la « souplesse », est vite devenu une réalité.

\section{NOTES}

1.Rapport d'activité du CNRS, octobre 1957-octobre 1958, p. 106.

2.Rapport d'activité du CNRS, octobre 1962-octobre 1963, p. 396.

3.Voir à ce sujet D. Guthleben, « Faut-il brûler le CNRS ? L'image du CNRS dans les médias ", in La Revue pour l'histoire du CNRS, $\mathrm{n}^{\circ}$ 9, novembre 2003, pp. 42-51.

4.Procès verbal de la séance du 29 octobre 1974 du conseil d'administration, p. 16, Archives du CNRS, Gif-sur-Yvette, G940035-24.

5.Ibid., p. 6.

6.Ibid., p. 17.

7.Procès verbal de la séance du 28 janvier 1975 du conseil d'administration, p. 8, Archives du CNRS, Gif-sur-Yvette, G940035-24.

8.Extrait d'une intervention effectuée à la séance du 23 mars 1987 du séminaire pour le cinquantenaire du CNRS.

9.Voir à ce sujet $\mathrm{G}$. Ramunni, Les sciences pour l'ingénieur. Histoire du rendez-vous des sciences et de la société, Paris, Éditions du CNRS, 1995. 
10.Procès verbal de la séance du 30 juin au 2 juillet 1975 du conseil directoire, pp. 23-28, Archives du CNRS, Gif-sur-Yvette, G940035 IHTP-3.

11.Ibid., p. 27.

12.Procès verbal de la séance du 10 juillet 1978 du conseil d'administration, p. 10,

Archives du CNRS, Gif-sur-Yvette, G940035-24.

\section{RÉSUMÉS}

En juillet 1975, le CNRS lance le PIRDES, programme interdisciplinaire de recherche pour le développement de l'énergie solaire. Le Centre répond à une demande pressante: les conséquences du choc pétrolier survenu deux ans plus tôt poussent à « la mobilisation de tout le potentiel scientifique et technique du pays dans ce domaine ».

In July 1975, the CNRS launched the PIRDES, an interdisciplinary research program for the development of solar energy. The PIRDES responded to a pressing request: the mobilization of the French scientific and technical potential in order to fight the consequences of the oil crisis.

INDEX

Mots-clés : programme, PIRDES, solaire, énergie solaire

\section{AUTEUR}

\section{DENIS GUTHLEBEN}

Denis Guthleben, historien, est attaché scientifique au Comité pour l'histoire du CNRS. 